\title{
CR Research Soure

\section{A Country Level Analysis Comparing Hospital Capacity and Utilisation During the First COVID-19 Wave Across Europe}

Elke Berger ( $\sim$ elke.berger@tu-berlin.de )

Technische Universität Berlin https://orcid.org/0000-0002-1932-6217

Juliane Winkelmann

Technische Universität Berlin: Technische Universitat Berlin

Helene Eckhardt

Technische Universität Berlin: Technische Universitat Berlin

Ulrike Nimptsch

Technische Universität Berlin: Technische Universitat Berlin

Dimitra Panteli

Technische Universität Berlin: Technische Universitat Berlin

Christoph Reichebner

Technische Universität Berlin: Technische Universitat Berlin

\section{Tanja Rombey}

Technische Universität Berlin: Technische Universitat Berlin

\section{Reinhard Busse}

Technische Universität Berlin: Technische Universitat Berlin

\section{Research article}

Keywords: COVID-19, hospital capacity, hospital utilisation, intensive care, ICU surge capacity

Posted Date: February 19th, 2021

DOI: https://doi.org/10.21203/rs.3.rs-251815/v1

License: (c) (1) This work is licensed under a Creative Commons Attribution 4.0 International License. Read Full License

Version of Record: A version of this preprint was published at Health Policy on December 1st, 2021. See the published version at https://doi.org/10.1016/j.healthpol.2021.11.009. 


\section{Abstract}

Background: The exponential increase of SARS-CoV-2 infections during the first wave of the pandemic created an extraordinary overload and demand on hospitals, especially on intensive care units (ICUs), across Europe. European countries have taken different measures to surge ICU capacity, but little is known on the extent. A country level analysis was conducted to compare hospitalisation rates of COVID19 patients in acute and intensive care and the levels of surge capacity for intensive care beds across 16 European countries and Lombardy region during the first wave of the pandemic (28 February to 31 July).

Methods: We used data on infection rates and numbers of current and/or cumulative COVID-19 patients in acute and intensive care in 16 countries and Lombardy region to analyse the burden on hospitals during the first wave of the COVID-19 pandemic. Data on COVID-19 hospitalisations was continuously extracted since 20 March, 2020 from publicly available sources. To evaluate whether hospital capacities were exceeded, we retrieved information on hospital and ICU surge capacity. Treatment days and mean length of hospital stay were calculated to assess hospital utilisation by COVID-19 patients during the first wave.

Results: Pre-pandemic hospital and ICU capacity varied widely across countries. In no studied country did the utilisation of acute care bed capacity by COVID-19 patients exceed $38.3 \%$. However, the Netherlands, Sweden, and Lombardy would not have been able to treat all COVID-19 patients during the first wave without ICU surge capacity. Indicators of hospital utilisation were not consistently related to the numbers of SARS-CoV-2 infections. The mean number of hospital days associated with one SARS-CoV-2 case ranged from 1.3 (Norway) to 11.8 (France).

Conclusion: In many countries, the increase of ICU capacity was important to accommodate the high demand for intensive care during the first COVID-19 wave. Our study indicates that SARS-CoV-2 incidence is not the only aspect when it comes to the burden of hospital care for COVID-19 but rather the utilisation of hospital resources as shown by cumulative hospital days and mean length of stay during the first wave. Indicators presented in this study could inform forecasting models, especially in regard to necessary surge capacity.

\section{Background}

Health systems have faced substantial pressures related to the COVID-19 pandemic. The exponential increase of SARS-CoV-2 infections in March and April, 2020, created an unprecedented demand on hospitals in many European countries. On March 11, the bed occupancy in some hospitals in Italy exceeded existing intensive care unit (ICU) capacities [1]. Shortly after, in late March, many Spanish hospitals, especially in the capital city of Madrid, reported reaching their capacity limits of acute and/or intensive care beds to adequately treat all COVID-19 patients requiring inpatient care [2]. Many hospitals in other European regions (e.g. the Dutch region of Brabant and Grand Est in France) were also overwhelmed with the influx of COVID-19 patients and transferred critically-ill patients to other hospitals 
across the country or even neighbouring countries to free up capacity [3]. During the second wave starting in autumn 2020, many countries face again rising hospital admission rates and substantial pressure on hospital wards.

While recognising that many health systems have, at least in part, been overburdened during the first wave, still little is known on the extent of hospital surge capacity created in acute and intensive care as a response to COVID-19 accommodated the spike of patients and the actual use of hospital capacities across European countries. However, to inform pandemic preparedness and contingency planning within the hospital setting, information on capacities, both in terms of initial and reserve surge capacities, as well as resource utilisation of COVID-19 patients is key [4]. Until the time of writing, existing studies only report on hospital surge capacities in single countries [5] or hospitals [6]; to the best of our knowledge there is no cross-country overview of additional surge capacity for COVID-19 patients. Furthermore, evidence on COVID-19 patients' length of stay in acute and intensive care is available only for a few European countries [7-9].

This paper aims to analyse whether COVID-19 hospitalisations exceeded national ICU surge capacity across 16 European countries and the Lombardy region during the first wave of the pandemic (28 February to 31 July). We complement this analysis with a comparison of the average length of stay and the cumulative hospital days of COVID-19 patients across the countries. The latter is additionally compared to the rate of SARS-CoV-2 infections. The findings aim to support health care decision makers in refining contingency plans and improving hospital preparedness for future health emergencies.

\section{Methods}

\section{Data collection and ethical considerations}

This cross-country analysis is based on a broader data collection initiative encompassing data on 18 European countries and two Italian regions. Countries were included in the data collection of hospitalised COVID-19 patients in acute and intensive care, which was kicked off on 20 March, 2020, once they reported at least five positive cases per 100,000 population. Data stems from official online sources such as websites of ministries of health, national research and public health institutes, official dashboards from national institutions and Our World in Data (OWiD) [10]. The resulting database has been updated daily since, and is made publicly available on the website of our institution [11] and the Harvard Dataverse repository [12]. All authors were involved in the daily updates of data. The study only relies on aggregated data that is publicly available; hence it does not contain individual level data and therefore ethical considerations are not applicable.

Furthermore, the database was updated regularly to reflect values that some countries corrected retrospectively (e.g. Norway, United Kingdom [UK]) (see supplemental tables 1-18, appendix pp 1-23). Over time, some countries adjusted their data collection method, data reporting channel or the style of reporting (i.e. Norway). If updated data was available retrospectively, we adjusted the collected data in 
our database accordingly. The ECDC started to provide similar data on current hospital and ICU occupancy for COVID-19 in several European countries as of summer 2020 [13].

Moreover, we retrieved information on the numbers of hospital beds prior to the pandemic from OECD [14] and ICU beds prior to the pandemic from OECD/European Union [15]. Information on surge capacity of ICU beds during the first wave was obtained from the COVID-19 Health System Response Monitor (HRSM) [16], with some supplementary information from national sources. The HSRM tool was established in March, 2020, and designed in response to the COVID-19 outbreak to collect and disseminate up-to-date information on how countries, mainly in the WHO European Region, are responding to the crisis, focused primarily on the responses of health systems.

\section{Data description}

For the data collected on hospitalised patients and patients in intensive care, definitions and reported units differ between countries due to different data collection methods (see supplemental tables 1-18, appendix pp 1-23). For most countries, information whether hospitalised COVID-19 cases represent confirmed cases only, or confirmed and suspected cases combined is not available. Where both confirmed and suspected cases were reported, we only retrieved data on confirmed cases. Furthermore, data collection varies in regard to reason and trajectory of hospital admission, namely whether patients were admitted only due to COVID-19 (and excluding other pathologies as well as transfers from other hospitals, i.e. in Belgium) or comprise all hospitalised cases that tested positive for COVID-19 (i.e. in Denmark and France). Moreover, some countries subsumed ICU patients in the total number of hospitalised patients, while others did not (e.g. the Netherlands). To calculate the number of all currently hospitalised patients we added up the numbers of inpatients treated in normal wards and in ICUs. The definition of ICU cases is unclear in some countries, e.g. regarding the inclusion of patients in surveillance beds. A detailed description of collected data, including sources, definitions, first or last date of reporting, and collection method for each country (and region) can be found in the supplemental tables 1-18, appendix pp 1-23.

\section{Analysis}

The presented analysis focuses on a subset of 16 European countries and one Italian region, for which information on current hospitalisations and/or ICU treatments of COVID-19 patients for the period until 31 July, 2020, was available, i.e. Austria, Belgium, Denmark, Estonia, Finland, France, Germany, Greece, Ireland, Italy (and the Lombardy region), Luxembourg, Netherlands, Norway, Portugal, Sweden, and UK. Spain and Switzerland did not report on COVID-19 patients currently treated in acute and intensive care and were therefore excluded from the analysis. Lombardy region was included as it had the highest number of cases of COVID-19 in Europe and in the world during the early months of 2020 [17]. To allow for a comparative analysis also for countries with incomplete daily reporting (e.g. no data on weekends), single missing values for currently hospitalised patients were imputed linearly.

First, we present and compare hospital and ICU bed capacities prior to COVID-19 in 14 countries and Lombardy region. In addition, we depict the additional ICU beds that were available during the surge for 
the COVID-19 pandemic in 11 countries as reported in the HSRM and national sources.

Second, to analyse whether hospital capacities were exceeded, we plotted the number of currently hospitalised COVID-19 patients per 100,000 population over time against the hospital bed capacities before the pandemic and grouped countries with similar capacity levels. We followed the same approach for ICU bed capacities, with an additional step of illustrating ICU surge capacity against the current hospitalisation rates of COVID-19 patients in intensive care. Due to limited data availability, 11 countries and Lombardy region were included in the analysis of acute bed capacities and nine countries and the Lombardy region in the analysis of ICU surge capacities.

We calculated additional indicators to further illustrate COVID-19 related hospital utilisation. Cumulative hospital days and cumulative ICU days were calculated by summing up the respective daily numbers of current inpatients (representing the total number of bed occupancy days until 31 July, 2020). For intensive care, this was done for all 16 countries plus the Lombardy region. With respect to acute care, Greece and Sweden were excluded due to lack of data. The relation between bed occupancy days and burden of SARS-CoV-2 infections was expressed as the mean number of hospital days, and ICU days per infected case. The mean length of stay was approximated for hospital and ICU treatment by dividing the number of bed occupancy days by the cumulative number of hospital and ICU treatment cases, respectively; this could be done for eight countries for acute care and for six countries for intensive care. Finally, we calculated the proportion of cumulative SARS-CoV-2 cases with hospital treatment and with ICU treatment for the same countries. Supplemental table 19 (appendix, p 24) provides an overview of countries included in each analysis.

\section{Results}

The pre-pandemic hospital and ICU capacity varied widely across the 14 countries with data available ranging from 197 acute care beds per 100,000 in Sweden to 602 beds in Germany[14] and from 5.0 ICU beds per 100,000 in Ireland (2016) and Sweden[15] to 33.4 ICU beds in Germany (2018)[18] (see Table 1). There is thus a three-fold variation for acute care capacity and even a seven-fold variation for intensive care bed capacity between the two ends of the spectrum in these countries prior to the COVID-19 pandemic. 
Table 1

Pre-pandemic hospital capacity, ICU capacity and ICU surge capacity during the first COVID-19 wave

\begin{tabular}{|c|c|c|c|c|c|c|c|}
\hline \multirow[t]{2}{*}{$\begin{array}{l}\text { Country/ } \\
\text { region }\end{array}$} & \multicolumn{2}{|c|}{$\begin{array}{l}\text { Acute care capacity } \\
\text { bed prior to COVID-19 } \\
\text { (2018) [14] }\end{array}$} & \multicolumn{2}{|c|}{$\begin{array}{l}\text { ICU bed capacity } \\
\text { prior to COVID-19 }\end{array}$} & \multirow[t]{2}{*}{ Year } & \multicolumn{2}{|c|}{$\begin{array}{l}\text { ICU bed surge capacity } \\
\text { during the first wave of } \\
\text { COVID-19 }\end{array}$} \\
\hline & in total & $\begin{array}{l}\text { per } \\
100,000\end{array}$ & in total & $\begin{array}{l}\text { per } \\
100,000\end{array}$ & & in total & $\begin{array}{l}\text { per } \\
100,000\end{array}$ \\
\hline Austria & 47,276 & 535 & $2500^{a}$ & $\begin{array}{l}28.9 \\
{[15]}\end{array}$ & 2018 & $\mathrm{n} / \mathrm{a}$ & $\mathrm{n} / \mathrm{a}$ \\
\hline Belgium & 56,758 & 497 & $1993^{a}$ & $\begin{array}{l}17.4 \\
{[15]}\end{array}$ & 2019 & $\begin{array}{l}\text { ca. } 2750 \\
\text { (early April) }^{\text {a }}\end{array}$ & 24 [16] \\
\hline Denmark & 13,659 & 236 & $\begin{array}{l}1078^{b} \\
{[19]}\end{array}$ & $18.6^{c}$ & 2020 & $\begin{array}{l}1242(925 \text { for } \\
\text { COVID-19 } \\
\text { patients) [19] }\end{array}$ & $21.4^{\mathrm{C}}$ \\
\hline Estonia & 4444 & 336 & $200^{a}$ & $\begin{array}{l}15.0 \\
{[15]}\end{array}$ & 2019 & $\begin{array}{l}130 \text { for } \\
\text { COVID-19 } \\
\text { patients }^{d}[16]\end{array}$ & $\mathrm{n} / \mathrm{a}$ \\
\hline Finland & 15,667 & 284 & $300^{\mathrm{a}}$ & $5.4[15]$ & 2019 & $\mathrm{n} / \mathrm{a}$ & $\mathrm{n} / \mathrm{a}$ \\
\hline France & 203,662 & 304 & $10,923^{a}$ & $\begin{array}{l}16.3 \\
{[15]}\end{array}$ & 2018 & $\mathrm{n} / \mathrm{a}$ & $\mathrm{n} / \mathrm{a}$ \\
\hline Germany & 497,182 & 602 & $\begin{array}{l}27,463 \\
{[18]}\end{array}$ & $33.4^{c}$ & 2018 & $\begin{array}{l}\text { 32,824 (early } \\
\text { June) [20] }\end{array}$ & $39.5^{c}$ \\
\hline Greece & 39,011 & 363 & $565^{a}$ & $5.3[15]$ & 2019 & $\begin{array}{l}1017 \text { (350 for } \\
\text { COVID-19 } \\
\text { patients, end } \\
\text { of April) [16] }\end{array}$ & $9.5^{c}$ \\
\hline Ireland & 13,560 & 279 & $250^{a}$ & $5.0[15]$ & 2016 & $\begin{array}{l}489 \text { (end of } \\
\text { May)/up to } \\
800[21,22]\end{array}$ & $10.0 / 16.3^{c}$ \\
\hline Italy & 156,216 & 259 & $5190^{a}$ & 8.6 [15] & 2020 & 8550 [23] & $14.0^{c}$ \\
\hline Lombardy & $\begin{array}{l}34,756 \\
{[24]}\end{array}$ & 346 [24] & $\begin{array}{l}724 \\
{[25]}\end{array}$ & $7.2^{c}$ & 2019 & $1347^{e}[16]$ & $13.4^{\mathrm{C}}$ \\
\hline Luxembourg & 2251 & 370 & $\mathrm{n} / \mathrm{a}$ & $\mathrm{n} / \mathrm{a}$ & $\mathrm{n} / \mathrm{a}$ & $\mathrm{n} / \mathrm{a}$ & $\mathrm{n} / \mathrm{a}$ \\
\hline Netherlands & 46,323 & 269 & $1150^{a}$ & $6.7[15]$ & 2018 & $\begin{array}{l}2400 \text { (1 } 900 \\
\text { for COVID-19 } \\
\text { patients) [26] }\end{array}$ & 13.9 \\
\hline Norway & 16,646 & 313 & 450 & 8.5 [15] & 2018 & $\begin{array}{l}\text { up to } 925 \text { (or } \\
\text { even } 1200^{f} \text { ) } \\
\text { [16] }\end{array}$ & $\begin{array}{l}17.4^{\mathrm{c}} \\
\left(22.5^{\mathrm{c}}\right)\end{array}$ \\
\hline
\end{tabular}




\begin{tabular}{|llllllll|}
\hline $\begin{array}{l}\text { Country/ } \\
\text { region }\end{array}$ & $\begin{array}{l}\text { Acute care capacity } \\
\text { bed prior to COVID-19 } \\
\text { (2018) [14] }\end{array}$ & $\begin{array}{l}\text { ICU bed capacity } \\
\text { prior to COVID-19 }\end{array}$ & Year & \multicolumn{2}{l|}{$\begin{array}{l}\text { ICU bed surge capacity } \\
\text { during the first wave of } \\
\text { COVID-19 }\end{array}$} & \\
\hline Portugal & 33,850 & 329 & $\begin{array}{l}587 \\
{[16]}\end{array}$ & $5.7^{c}$ & n/a & n/a & n/a \\
\hline Sweden & 20,019 & 197 & $\begin{array}{l}526 \\
{[16]}\end{array}$ & $5.0^{c}$ & 2020 & $\begin{array}{l}1064 \text { (mid- } \\
\text { April) }[16]\end{array}$ & $10.4^{c}$ \\
\hline UK & $n / a$ & $n / a$ & $n / a$ & $n / a$ & $n / a$ & $n / a$ & $n / a$ \\
\hline
\end{tabular}

Notes: a: Own calculation based on ICU beds prior to COVID-19 per 100,000; b: Including 645 ICU beds usually reserved for patients with elective surgery; c: Own calculation based on ICU beds prior to COVID19 in total; $d$ : It is unclear, whether these beds were created additionally or reserved to patients with COVID; e: Calculation based on information from HSRM that ICU bed capacity increased by $86 \%$; $f$ : On April 15th the Regional Health Authorities provided detailed contingency plans on how to increase the ICU capacity to 1200 beds, but underlined that such a capacity can only be sustained for a short period of time.

Figure 1 further illustrates the association between acute care and ICU bed capacity for nine countries and the Lombardy region: countries with high acute bed capacity tend to also have high ICU bed capacity. Denmark seems to be an exception, with relatively high ICU bed capacities compared to lower acute hospital bed capacity.

\section{Sources}

Acute care and ICU beds prior to COVID-19 pandemic [14] [15] and ICU beds during COVID-19 [16]; see Table 1 for more information.

\section{Notes}

BE - Belgium, DE - Germany, DK - Denmark, GR - Greece, IE - Ireland, IT - Italy, IT-25 - Lombardy, NLThe Netherlands, NO - Norway.

In response to the rapidly rising numbers of COVID-19 patients, countries have implemented different strategies such as postponement of elective surgery or the setup of field hospitals to rapidly create additional acute and ICU beds [27]. Some countries that did not see a critical increase of COVID-19 cases and hospitalisations, such as Denmark, Estonia, and Norway, had contingency plans in place to reserve surge capacity for extreme situations.

Figure 1 shows the number of ICU beds per 100,000 additionally created (or planned) during the first wave, based on information reported in the COVID-19 HSRM and national sources (see also Table 1). In Italy's hard-hit Lombardy region ("IT-25" in Fig. 1), intensive care capacity nearly doubled since the start of the COVID-19 crisis. Numbers of ICU beds were also significantly increased in Belgium, Germany, Greece, Ireland, Italy, the Netherlands, and Sweden. According to Norway's contingency plan, ICU capacity could 
be more than doubled in an emergency situation. A similar increase of beds likely occurred in acute care, but data was not consistently reported in the HSRM and country specific sources.

Figure 2A illustrates daily rates of hospitalised COVID-19 patients in regard to total available acute hospital beds in 11 countries. It shows that pre-pandemic capacity of acute care beds was not exceeded by COVID-19 hospitalisations in any of the included countries. In Lombardy region, the percentage of acute care beds occupied by COVID-19 patients peaked at $38.3 \%$, followed by Italy with $21.1 \%$, while in Austria the maximum was $2.3 \%$.

In contrast, Fig. 2B plotting pre-pandemic capacity and surge capacity of ICU beds against daily rates of COVID-19 patients in intensive care shows that $\mathrm{i}$ intensive care capacity prior to the pandemic was exceeded by COVID-19-necessitated admissions in the Netherlands, Sweden, and the Lombardy region, and that capacities were nearly surpassed in Belgium and Italy. In Lombardy and Sweden, for around five weeks the demand for intensive care by COVID-19 patients exceeded pre-pandemic capacities, while the shortage lasted around two weeks in the Netherlands. Only in Lombardy region surge capacity was fully exhausted and even exceeded for one day (on 3 April, 2020).

\section{Sources}

OECD [14], among others; see Table 1 for detailed information.

\section{Notes}

February to July, 2020; black line - current COVID-19 hospitalisations per 100,000; orange line - rate of hospital beds per 100,000.

\section{Sources}

ICU beds prior to COVID-19 pandemic - OECD/European Union [15], among others; ICU beds during COVID-19 - COVID-19 HSRM [16], among others; see Table 1 for detailed information.

\section{Notes}

black line - current COVID-19 hospitalisations in intensive care per 100,000 (February to July, 2020); red line - rate of ICU beds per 100,000 before the COVID-19 pandemic; red dashed line - rate of ICU beds per 100,000 during the COVID-19 pandemic.

Figure $3 \mathrm{~A}$ reports the total number of days COVID-19 patients spent in acute and intensive care per 100,000 population (countries are sorted by cumulative COVID-19 incidence until 31 July, 2020). The number of days COVID-19 patients were treated in acute and intensive care settings varied widely across countries; until 31 July, the cumulative number of hospital days per 100,000 inhabitants ranged from 210 in Finland to 7762 in Lombardy (Italy) and the cumulative number of days in intensive care from 38 in 
Greece to 678 in Lombardy, followed by France, Italy, and Belgium. For France, high numbers of hospital and ICU treatment days were observed, while the reported overall SARS-CoV-2 incidence was comparatively low. Other countries, like Ireland and Portugal, had a higher SARS-CoV-2 incidence but notably lower numbers of hospital and ICU treatment days. The proportion of cumulative days spent in intensive care in relation to cumulative days spent in normal wards ranged from $9 \%$ in Lombardy and Estonia up to $38 \%$ in the Netherlands.

\section{Notes}

The cumulative incidence considers all cases of SARS-CoV-2 up to July 31, 2020. Cumulative hospital days could not be calculated for Sweden and Greece as they did not report daily numbers of current hospitalisations. Norwegian data of current ICU treatments were only available until June 19. Cumulative SARS-CoV-2 incidence in Luxembourg includes cases among the resident and non-resident population.

Notes: 3B: Mean length of stay was approximated for hospital treatment by dividing the daily numbers of current hospitalisations (representing bed occupancy days) by the cumulative number of hospital cases. For Germany, actual mean length of stay was obtained from national hospital discharge data. 3C: Mean length of ICU stay was approximated by dividing the daily numbers of current ICU treatments (representing ICU bed occupancy days) by the cumulative number of ICU treatment cases. Norwegian data of current ICU treatments were only available until 19 June, 2020.

Figures 3B and 3C depict the share of COVID-19 patients treated in acute and intensive care settings, the mean lengths of stay and the mean number of hospital and ICU treatment days per SARS-CoV-2 case, with the latter determining the order of countries in the figures. The left side displays the number of COVID-19 patients treated in hospitals and ICUs as a share of all SARS-CoV-2 cases until 31 July and the right shows the mean lengths of hospital stay of COVID-19 patients.

The mean number of hospital days associated with one SARS-CoV-2 case ranged from 1.3 (Norway) to 11.8 (France). The proportion of all SARS-CoV-2 cases that received inpatient treatment ranged from $11 \%$ (Norway) to $57 \%$ (France). The mean length of hospital stay varied from 8 days (UK) to 21 days (France).

The mean number of ICU days associated with one SARS-CoV-2 case ranged from 0.3 (Ireland) to 1.1 (Netherlands). The proportion of SARS-CoV-2 cases with ICU treatment ranged from $1.7 \%$ (Ireland) to $5.4 \%$ (Germany and the Netherlands). The mean length of ICU stay varied from 13 days (Germany) to 21 days (UK).

\section{Discussion}

To the best of our knowledge, this study is the first cross-country overview of hospitalisations associated with COVID-19 patients, and additionally created intensive care resources. Our results indicate that the pre-pandemic hospital capacities varied widely between countries included in the analysis. Irrespective of their starting point, countries have taken several measures to boost ICU capacity during the first wave of 
the pandemic to meet the spike in demand for hospital care, although to a different extent. The highest increase of ICU beds was achieved in Ireland, the Netherlands, and Sweden where capacity doubled compared to pre-COVID levels. Lombardy region and Greece also increased their initial capacity of ICU beds by $86 \%$ and $80 \%$, and Italy and Belgium by $65 \%$ and $38 \%$, respectively. In contrast, Germany increased its ICU capacity only by $20 \%$ which is likely to be linked to its high initial capacity of ICU beds.

While countries faced different numbers of confirmed SARS-CoV-2 infections, available capacities of acute care beds were not exceeded by COVID-19 patients in the included countries. However, our analysis shows that the Netherlands and Sweden would not have had sufficient capacity to treat all COVID-19 patients requiring intensive care without ICU surge capacity. In the Lombardy region ICU surge capacity was even exceeded.

Our calculations of hospital indicators showed that COVID-19-related hospital utilisation varied widely across the countries included. We observed a large difference of the number of cumulative treatment days of COVID-19 patients in acute and intensive care settings and could not reveal a consistent relation to the incidence ofSARS-CoV-2. This suggests that SARS-CoV-2 incidence alone is not the driving force for the utilisation of acute and intensive hospital care but many other factors such as demographics of SARS-CoV-2 cases and testing strategies may play an important role. For example, the true number of persons infected with SARS-CoV-2 might be underestimated, depending on different testing strategies across countries [28].

Consequently, the mean number of hospital days per SARS-CoV-2 case varied from 1.3 (Norway) to 11.8 (France), and the number of ICU days per infected case varied from 0.3 (Ireland) to 1.1 (Netherlands). These figures may serve as basic landmarks for forecasting capacity requirements to meet surge demand. In Belgium, for example, based on the data from March to July, 2020, 1000 additional infections would cause on average a need of 3800 hospital bed days (mean days per case 3.8) and 800 ICU bed days (mean days per case 0.80 ).

The study has several limitations, which should be acknowledged, before discussing its usefulness e.g. for forecasting hospital capacity requirements. First, information on the timing of surge capacity in a country, including when it started, how quickly it scaled up, and if/when it scaled down, is uncertain. This is related to the fact that the level of information reported in the HSRM is not systematically harmonised across countries. Second, there are some uncertainties regarding data quality and data completeness within countries, both related to data on hospital utilisation and information reported on HSRM. Third, non-COVID-19 patients requiring acute or intensive care were not included in our analysis, which should be taken into consideration to comprehensively esteem the full burden on hospitals during the first wave. Fourth, with the exception of the Lombardy region, this study is a country-level analysis and does not consider the geographic distribution of hospital capacity, COVID-19 admissions and patterns of hospital utilisation; there might have been periods of time where ICUs were overloaded in certain regions within a country which are not reflected by our data. We show that this was the case for Lombardy, while it was not the case for Italy as a whole. Along that line our data does not allow to identify whether single 
hospitals' capacities were exceeded. Mateen et al. [29], for example, reported that hospital capacities of one third of all hospitals in England were exceeded during the first COVID-19 wave. Fifth, the focus of our study is on hospital bed capacities without considering capacities of health professionals and medical equipment such as PPE (personal protective equipment) and ventilators that are necessary to keep surge capacities for COVID-19 patients operational. Any decision on planning capacity should take these aspects into account [4]. Sixth, there is no disaggregation both on data related to intensive care capacity and utilisation, i.e. low, high or intermediate care. Hence, there may be differences in the notion of intensive care influencing the comparability of the data. Further, the informative value of our data in regard to length of hospital stay of COVID-19 patients is limited, as we could only approximate the mean length of stay instead of calculating the median which was reported in the majority of existing studies [7, 8]. Last, comparability of data across countries is limited, e.g., due to different data collection methods and definitions of variables, such as hospitalised COVID-19 patients, which in some countries also include unconfirmed cases, or the numbers of SARS-CoV-2 cases (in some countries confirmed only by PCR tests, in others also by antibody tests, see supplemental tables 1-18, appendix pp 1-23).

However, when comparing our data to published literature our findings appear to be plausible. For example, the Norwegian ICU registry reports an average length of stay of 17 days in October, 2020 [30], which is similar to our data from 19 June (latest available date in our database) showing that patients spent on average 16 days in the ICU, with the variation likely related to the different time of measurement. Further, data from OECD/European Union [15] reported ICU occupancy levels of 78\% in Italy at the height of the outbreak, which is comparable with our data, where this proportion was about $80 \%$. However, they only relate the number of patients requiring intensive care to the initial number of ICU beds. Taking surge capacity into account, the number of ICU beds occupied by COVID-19 patients drops to $48 \%$. This shows the importance of surge capacity, which is highlighted in our analysis.

Further, the observed COVID-19-related hospital utilisation varied widely. Indicators of hospital utilisation, such as the share of hospitalisations among SARS-CoV-2 cases, or cumulative treatment days revealed no consistent relation with the number of persons infected with SARS-CoV-2. This suggests that SARSCoV-2 incidence alone is not decisive for the utilisation of acute and intensive hospital care. The true number of persons infected with SARS-CoV-2 might be underestimated to a greater or lesser extent, depending on different testing strategies across countries [28].

Consequently, the mean number of hospital days per SARS-CoV-2 case varied from 1.3 (Norway) to 11.8 (France), and the number of ICU days per infected case varied from 0.3 (Ireland) to 1.1 (Netherlands). These figures may serve as basic landmarks for forecasting capacity requirements to meet surge demand. In Belgium, for example, based on the data from March to July, 2020, 1000 additional infections would cause on average a need of 3800 hospital bed days (mean days per case 3.8) and 800 ICU bed days (mean days per case 0.80 ).

Data on available hospital resources combined with their utilisation is crucial to inform health care decision makers [31] over the course of the COVID-19 pandemic, but also for upcoming public health 
crises, e.g. by integrating indicators presented in this study into forecasting models. Finland, Norway, and Sweden [32], as well as the Netherlands and the UK (England, Wales, Northern Ireland) have a long tradition of ICU registries [33] and were therefore able to strictly monitor the daily situation during the pandemic, which is key for reacting in a timely manner. Other countries, such as Germany [20], set up ICU registries only after the pandemic had emerged.

Overall, European countries experienced hospital capacity utilisation differently. This underlines the importance of data collection and monitoring for planning authorities. Generally, being overprepared in extreme situations might be preferable over risking overwhelmed capacities. This is substantiated by recent work that indicates a higher mortality of COVID-19 patients in an area without access to intensive care [34].

\section{Conclusion}

Our study indicates that SARS-CoV-2 incidence is not the only aspect during the first COVID-19 wave when it comes to the burden of hospital care for COVID-19 but rather the utilisation of hospital resources as shown by cumulative hospital days and mean length of stay. Hospital resource utilisation also depends on demographics of infected population groups, treatment pathways, service delivery patterns including reimbursement policies, public health strategies and the number of hospital beds and ICU beds within a health system. The presented data might contribute to preparedness activities, such as forecasting surge capacity demand for the ongoing or a coming pandemic. In countries with low ICU capacities where pre-pandemic ICU capacities were exceeded, it might be useful to consider strategies for reserving ICU beds for future health emergencies. Upcoming studies on hospital utilisation during the second wave of the pandemic might add additional findings that will further contribute to preparedness activities, aiming to cope with future occurrences of unpredicted large-scale needs in acute health care.

\section{Abbreveations}

BE Belgium

DE Germany

DK Denmark

GR Greece

HSRM Health System Response Monitor

ICU Intensive Care Unit

IE Ireland

IT Italy

Page $12 / 18$ 
IT-25 Lombardy

NL The Netherlands

NO Norway

OWiD Our World in Data

PPE Personal Protective Equipment

UK United Kingdom

\section{Declarations}

Ethics approval and consent to participate: The study only relies on aggregated data that is publicly available; hence it does not contain individual level data and therefore ethical considerations are not applicable.

Consent for publication: Not applicable.

Availability of data and materials: The data that support this study was collected by the authors of the manuscript from multiple sources that are publicly available. The dataset generated and analysed during the current study are available in the Harvard Dataverse repository, accessible at https://doi.org/10.7910/DVN/02CFBB.

Competing interests: $E B, J W, H E, U N, C R, T R, D P$ and RB report grants from Ministry of Education and Research (BMBF) and the federal state of Berlin, during the conduct of the study. UN receives funding from the German Research Foundation. DP started working for the European Observatory on Health Systems and Policies on 1 October, 2020, a partnership hosted by the World Health Organization. The submitted work has been independent from this relationship. RB reports grants from Federal Ministry of Research and Education, grants from World Health Organization, grants and other from Federal Ministry of Health, grants from Innovation Fund, outside the submitted work.

Funding: The study was part of a project of the Berlin University Alliance, funded by the Ministry of Education and Research (BMBF) and the federal state of Berlin (112_PreEP_Corona). The funders of the study had no role in study design, data collection, data analysis, data interpretation, writing of the report, or the decision to submit the paper for publication.

Authors' contributions: EB, JW and RB conceived the study and its design. UN, HE, DP, CR and TR contributed ideas and feedback to the conceptualisation of the study. EB, JW, HE and UN contributed to data analysis and wrote the main draft of the manuscript. EB, JW, HE UN, CR, TR and DP contributed to acquisition and curation of data. RB and DP contributed to critical revision of the report and study supervision. All authors critically revised and approved the manuscript. 
Acknowledgments: Institutional support and physical resources were provided by the Technische Universität Berlin. Erin Webb was responsible for language editing and proof-reading.

\section{References}

1. Verelst F, Kuylen E, Beutels P. Indications for healthcare surge capacity in European countries facing an exponential increase in coronavirus disease (COVID-19) cases, March 2020. Euro Surveill 2020. doi:10.2807/1560-7917.ES.2020.25.13.2000323.

2. Jones S. Spanish minister says older people found 'dead and abandoned'. 23 March, 2020. https://www.theguardian.com/world/2020/mar/23/spain-distributes-650000-testing-kits-ascoronavirus-deaths-rise-steeply. Accessed 24 Nov 2020.

3. Winkelmann J, Scarpetti G, Hernandez-Quevedo C, van Ginneken E. How do the worst-hit regions manage COVID-19 patients when they have no spare capacity left? 2020. https://analysis.covid19healthsystem.org/index.php/2020/04/24/how-do-the-worst-hit-regionsmanage-covid-19-patients-when-they-have-no-spare-capacity-left/. Accessed 8 Dec 2020.

4. World Health Organization. Beyond Containment: Health systems responses to COVID-19 in the OECD; 2020.

5. McCabe R, Schmit N, Christen P, D'Aeth JC, Løchen A, Rizmie D, et al. Adapting hospital capacity to meet changing demands during the COVID-19 pandemic. BMC Med. 2020;18:329. doi:10.1186/s12916-020-01781-w.

6. Carenzo L, Costantini E, Greco M, Barra FL, Rendiniello V, Mainetti M, et al. Hospital surge capacity in a tertiary emergency referralcentre during the COVID-19 outbreak in Italy. Anaesthesia. 2020:928-34. doi:10.1111/anae.15072.

7. Lane EA, Barrett DJ, Casey M, McAloon CG, Collins ÁB, Hunt K, et al. Country differences in hospitalisation, length of stay and admission to Intensive Care Units due to SARS-CoV-2 infection: a rapid review of available literature (preprint). medRxiv 2020. doi:10.1101/2020.05.12.20099473.

8. Rees EM, Nightingale ES, Jafari Y, Waterlow NR, Clifford S, Pearson B. CA, et al. COVID-19 length of hospital stay: a systematic review and data synthesis. BMC Med. 2020;18:270. doi:10.1186/s12916020-01726-3.

9. Karagiannidis C, Mostert C, Hentschker C, Voshaar T, Malzahn J, Schillinger G, et al. Case characteristics, resource use, and outcomes of 10021 patients with COVID-19 admitted to 920 German hospitals: an observational study. The Lancet Respiratory Medicine. 2020. doi:10.1016/S2213-2600(20)30316-7.

10. Our World in Data (OWiD). Coronavirus Pandemic (COVID-19). 2020. https://ourworldindata.org/coronavirus. Accessed 18 Dec 2020.

11. Department of Health Care Management (MiG). COVID-19-STATS. 2020. https://www.mig.tuberlin.de/fileadmin/a38331600/sonstiges/COVID-19-STATS_2007_22.pdf. Accessed 18 Dec 2020. 
12. Winkelmann J, Berger E, Busse R, Nimptsch U, Reichebner C, Eckhardt H, et al. COVID-19 Hospitalisation, cases and tests in 18 European countries. 2021.

https://dataverse.harvard.edu/dataset.xhtml?persistentld=doi:10.7910/DVN/02CFBB. Accessed 28 Jan 2021.

13. European Centre for Disease Prevention and Control (ECDC). Data on hospital and ICU admission rates and current occupancy for COVID-19. 2021. https://www.ecdc.europa.eu/en/publicationsdata/download-data-hospital-and-icu-admission-rates-and-current-occupancy-covid-19. Accessed 26 Jan 2021.

14. OECD. Hospital beds; Curative (acute) care beds. 2020. https://stats.oecd.org/Index.aspx? ThemeTreeld=9\#. Accessed 26 Nov 2020.

15. OECD/European Union. Health at a Glance: Europe 2020: State of Health in the EU Cycle. Paris: OECD; 2020.

16. COVID-19 Health System Response Monitor (HSRM). COVID-19 Health System Response Monitor. 2020. https://www.covid19healthsystem.org/mainpage.aspx. Accessed 26 Nov 2020.

17. Cereda D, Tirani M, Rovida F, Demicheli V, Ajelli M, Poletti P, et al. The early phase of the COVID-19 outbreak in Lombardy, Italy. The Lancet. 2020;396:e86-7. doi:10.1016/S0140-6736(20)32154-1.

18. Statistisches Bundesamt. Grunddaten der Krankenhäuser: 2018; 2020.

19. Sundhedsstyrelsen. Håndtering af COVID-19: Prognose og kapacitet i Danmark for intensiv terapi; 2020.

20. Intensivregister DIVI. 2020. https://www.intensivregister.de/\#/index. Accessed 8 Dec 2020.

21. HSE. COVID19 Daily Operations Update. 2020. https://www.hse.ie/eng/services/news/newsfeatures/covid19-updates/covid19-daily-operationsupdate-2000-29-may-2020.pdf. Accessed 9 Dec 2020.

22. HSE. COVID-19 Committee Meeting Tuesday 2 June 2020. 2020.

https://www.hse.ie/eng/services/news/media/pressrel/covid-19-committee-meeting-tuesday-2-june2020.html. Accessed 9 Dec 2020.

23. Piantoni V. After years of cuts, Italy is set to double the number of ICU beds in response to Covid-19. 2020. https://www.lastampa.it/esteri/la-stampa-in-english/2020/05/07/news/after-years-of-cutsitaly-is-set-to-double-the-number-of-icu-beds-in-response-to-covid-19-1.38815375. Accessed 9 Dec 2020.

24. Eurostat. Hospital bed by NUTS2 regions. 2020.

https://ec.europa.eu/eurostat/databrowser/view/hlth_rs_bdsrg/default/table?lang=en. Accessed 18 Dec 2020.

25. Ministero della Salute. Open data. 2019.

http://www.dati.salute.gov.it/imgs/C_17_dataset_96_0_upFile.csv. Accessed 26 Nov 2020.

26. Lailor A. Number of ICU beds needed will decrease to normal levels by 1 May, OMT expects. 2020. https://dutchreview.com/news/number-of-icu-beds-needed-will-decrease-to-normal-levels-by-1-mayomt-expects/. Accessed 9 Dec 2020. 
27. Phua J, Weng L, Ling L, Egi M, Lim C-M, Divatia JV, et al. Intensive care management of coronavirus disease 2019 (COVID-19): challenges and recommendations. The Lancet Respiratory Medicine. 2020;8:506-17. doi:10.1016/S2213-2600(20)30161-2.

28. Raftery AE, Currie J, Bassett MT, Groves R. Evaluating Data Types: A Guide for Decision Makers using Data to Understand the Extent and Spread of COVID-19. Washington, D.C.: National Academies Press; 2020.

29. Mateen BA, Wilde H, Dennis Jm, Duncan A, Thomas NJM, McGovern AP, et al. A geotemporal survey of hospital bed saturation across England during the first wave of the COVID-19 Pandemic (preprint). medRxiv 2020. doi:10.1101/2020.06.24.20139048.

30. Borgan E. Norwegian COVID-19 patients: Physicians surprised by length of stay on ventilators. 2020. https://sciencenorway.no/covid19-epidemic-medical-procedures/norwegian-covid-19-patientsphysicians-surprised-by-length-of-stay-on-ventilators/1753844. Accessed 25 Nov 2020.

31. World Health Organization. Critical preparedness, readiness and response actions for COVID-19. 2020. https://apps.who.int/iris/rest/bitstreams/1283590/retrieve. Accessed 9 Dec 2020.

32. Strand K, Walther SM, Reinikainen M, Ala-Kokko T, Nolin T, Martner J, et al. Variations in the length of stay of intensive care unit nonsurvivors in three Scandinavian countries. Crit Care. 2010;14:R175. doi:10.1186/cc9279.

33. Walther SM, Karlström G. National ICU registries. In: Guidet B, Valentin A, Flaatten H, editors. Quality Management in Intensive Care: A Practical Guide: Cambridge University Press; 2016. p. 195-203.

34. Bauer J, Brüggmann D, Klingelhöfer D, Maier W, Schwettmann L, Weiss DJ, Groneberg DA. Access to intensive care in 14 European countries: a spatial analysis of intensive care need and capacity in the light of COVID-19. Intensive Care Med 2020. doi:10.1007/s00134-020-06229-6.

\section{Figures}




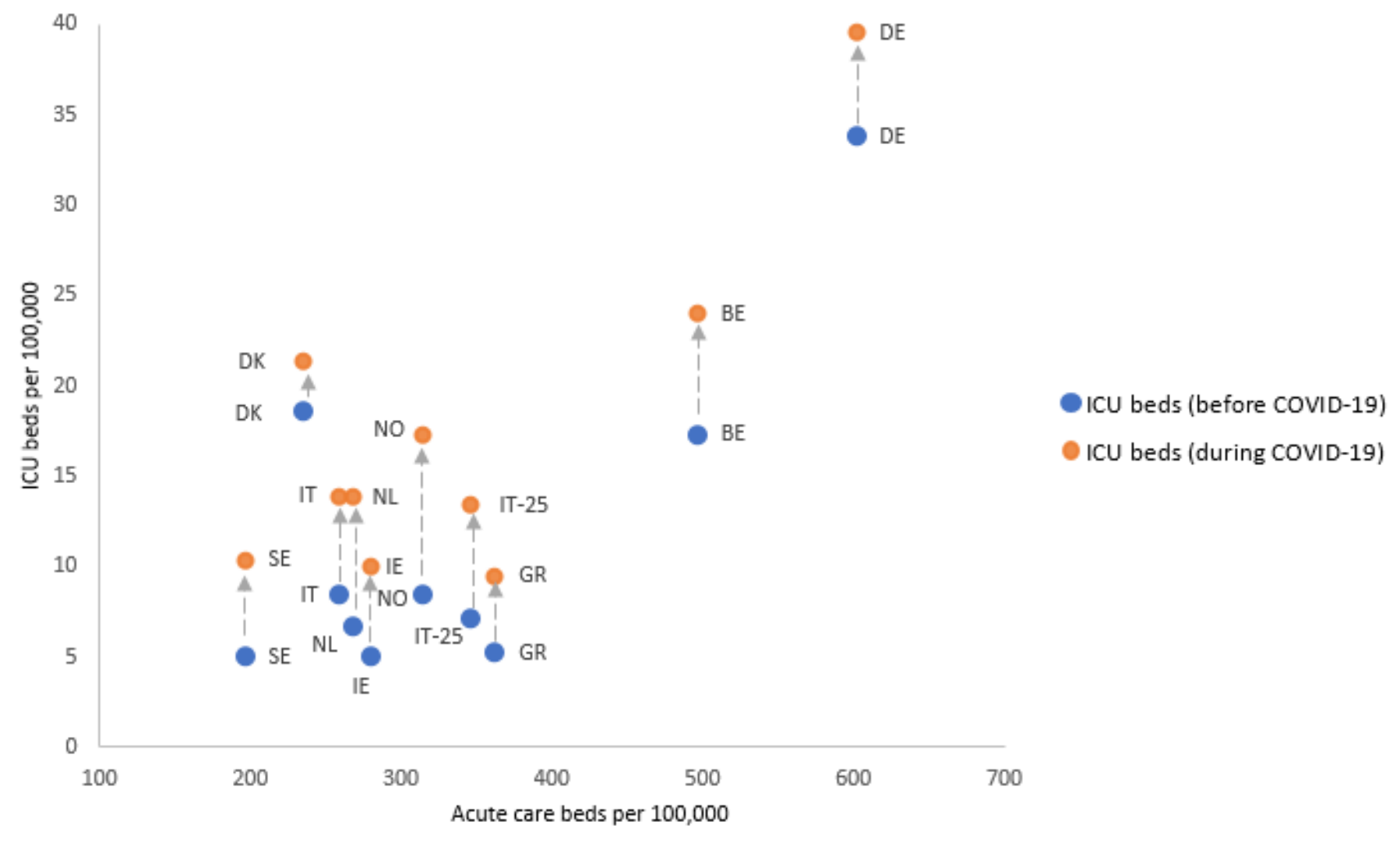

\section{Figure 1}

Hospital and ICU capacity before COVID-19 and ICU surge capacity created for COVID-19 (per 100,000)
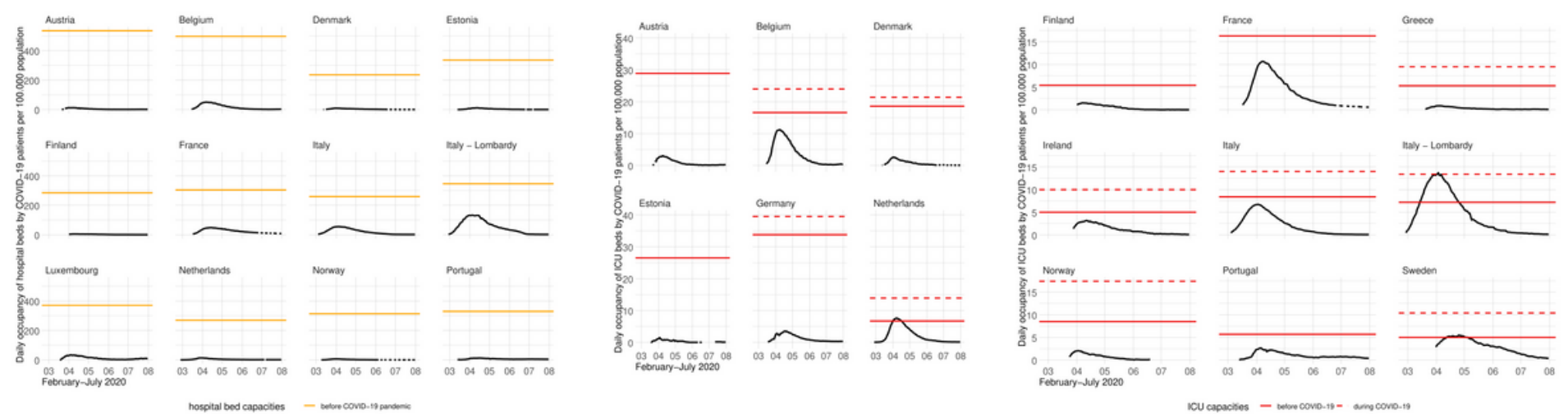

Figure 2

A Occupancy of hospitalised COVID-19 patients and pre-pandemic hospital bed capacity (per 100,000) B Occupancy of COVID-19 patients in ICU and pre-pandemic and surge ICU capacity created for COVID-19 (per 100,000) 


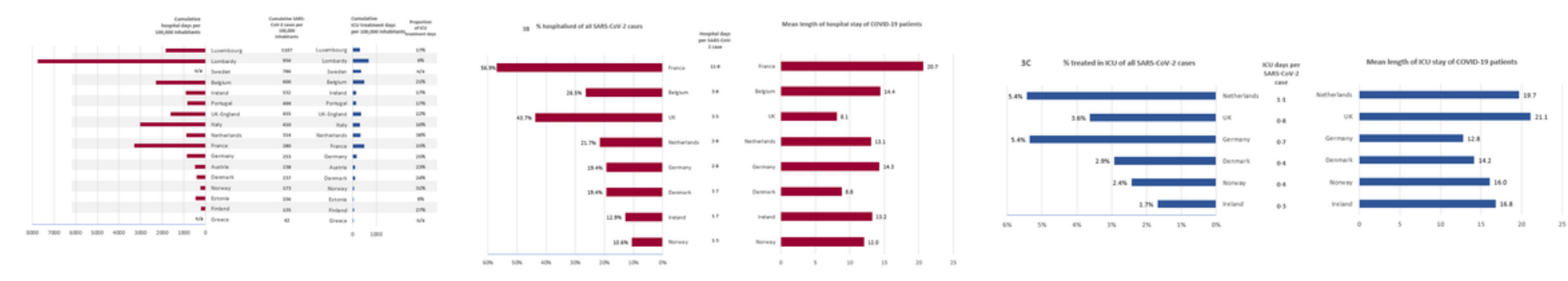

Figure 3

A Cumulative hospital days and ICU days of COVID-19 patients (per 100,000) B and Figure 3C Indicators on hospital utilisation of COVID-19 patients in acute and intensive

\section{Supplementary Files}

This is a list of supplementary files associated with this preprint. Click to download.

- SupplementalTable119.pdf 